\title{
A case of myeloproliferative neoplasm with a normal complete blood cell count: A novel problem of the JAK2 era
}

\author{
XIU-PENG YE, SHEN BAO, HUAN-MIN GAO, YING GUO and YU-PING WEI \\ Department of Hematology-Oncology, Ningxia People's Hospital, Yinchuan, Ningxia 750002, P.R. China \\ Received October 16, 2014; Accepted July 9, 2015
}

DOI: $10.3892 / 01.2016 .4192$

\begin{abstract}
The present study reported a case of a myeloproliferative neoplasm (MPN) in a patient with a normal complete blood cell count. Bone marrow biopsy showed bone marrow hyperplasia, an elevated megakaryocyte count, megakaryocytic dysplasia and pleomorphic changes, multiple megakaryocyte clusters and focal reticulin fiber hyperplasia. Furthermore, genetic analysis revealed that the patient was positive for the JAK2-V617F mutation, and negative for the JAK2 exon 12 and 13 mutations and the BCR-ABL (p210) fusion gene. The patient's condition was basically stable and at the time of writing, the patient remained in a stable condition with no specific symptoms of disease. The present study also analyzed the diagnostic and clinical features of MPNs, and a literature review was performed. MPN with a normal complete blood cell count is a rare disease, and attention should be focused on this entity in the clinic.
\end{abstract}

\section{Introduction}

Myeloproliferative neoplasms (MPNs) occur as a result of continuous clonal proliferation of one or a multiple series of mature and differentiated bone marrow cells (1). MPNs are characterized by an increased number of bone marrow nucleated cells, terminal differentiation and mature proliferative cells, and frequently occur without dysplasia (2). These neoplasms usually exhibit an insidious onset, with one or a multiple series of elevated peripheral blood cells, often accompanied by hepatosplenomegaly. Myelofibrosis, bone marrow failure and the development of acute leukemia may also occur in the later stages of the disease (3). Undifferentiated MPN accounts for $10-20 \%$ of all MPN cases, and is characterized by hepatosplenomegaly, leukocytosis, thrombocytosis and not having the Philadelphia chromosome or breakpoint cluster region protein-Abelson murine leukemia viral oncogene homolog 1 fusion gene $(4,5)$. Patients with undifferentiated

Correspondence to: Dr Shen Bao, Department of HematologyOncology, Ningxia People's Hospital, 301 Zhengyuan North Street, Yinchuan, Ningxia 750002, P.R. China

E-mail: yxp4200338@163.com

Key words: myeloproliferative neoplasms, JAK2 gene
MPN may or may not have anemia or an elevated red blood cell count. These characteristics do not conform to any diagnostic criteria; therefore, it is challenging to diagnose.

The reported incidence of MPNs worldwide is $\sim 0.1-3$ cases per 100,000 individuals, with no significant differences observed between regions and countries (6). The peak age of incidence is 50-60 years old (6). Untreated MPN patients exhibit a poor prognosis, and usually succumb within a few months following diagnosis (7). However, with the appropriate treatment certain patients may survive for a number of years. The application of molecular targeted drugs for MPN treatment may improve patient survival. Interferon (IFN)- $\alpha$ specifically blocks the JAK2V617F mutation of hematopoietic stem cells and may prevent the development of MPN (8). Previous studies concerning IFN- $\alpha$ treatment of MPN patients demonstrate that IFN- $\alpha$ may completely alleviate the hematology and molecular biology associated with MPN patients $(8,9)$.

The present study reported a case of a myeloproliferative neoplasm (MPN) in a patient with a normal complete blood cell count. The patient's condition was basically stable. The present study also analyzed the diagnostic and clinical features of MPNs, and a literature review was performed. MPN with a normal complete blood cell count is a rare disease, and attention should be focused on this entity in the clinic.

\section{Case report}

A 38-year-old female presented at Ningxia People's Hospital (Yinchuan, Ningxia, China) in April 2013 with intermittent splenomegaly, which was observed using ultrasound during an annual health-check. A physical examination revealed an enlarged spleen, but the liver was normal. Abdominal computed tomography also identified splenomegaly. A gastroscopic examination revealed gastric varices and multiple duodenal ulcers. Blood routine tests showed a white blood cell count of $9.71 \times 10^{9} / 1$ (normal range, 4.0-10.0× $10^{9} / 1$ ), an absolute neutrophil count of $6.82 \times 10^{9} / 1$ (normal range, $2.0-7.0 \times 10^{9} / 1$ ), a monocyte count of $0.38 \times 10^{9} / 1$ (normal range, $0.1-0.6 \times 10^{9} / 1$ ), an eosinophil count of $0.2 \times 10^{9} / 1$ (normal range, $0.02-0.52 \times 10^{9} / 1$ ), a red blood cell count of $4.5 \times 10^{12} / 1$ (normal range, 3.5-5.5 $\times 10^{12} / 1$ ), a hemoglobin level of $154 \mathrm{~g} / \mathrm{l}$ (normal range, 110-160 g/dl) and a platelet count of $208 \times 10^{9} / 1$ (normal range, 100-300 $\times 10^{9} / 1$ ). Markers for hepatitis B were negative and liver function tests were normal. Tests for tumor biomarkers were also normal. A bone marrow biopsy showed overactive bone marrow 


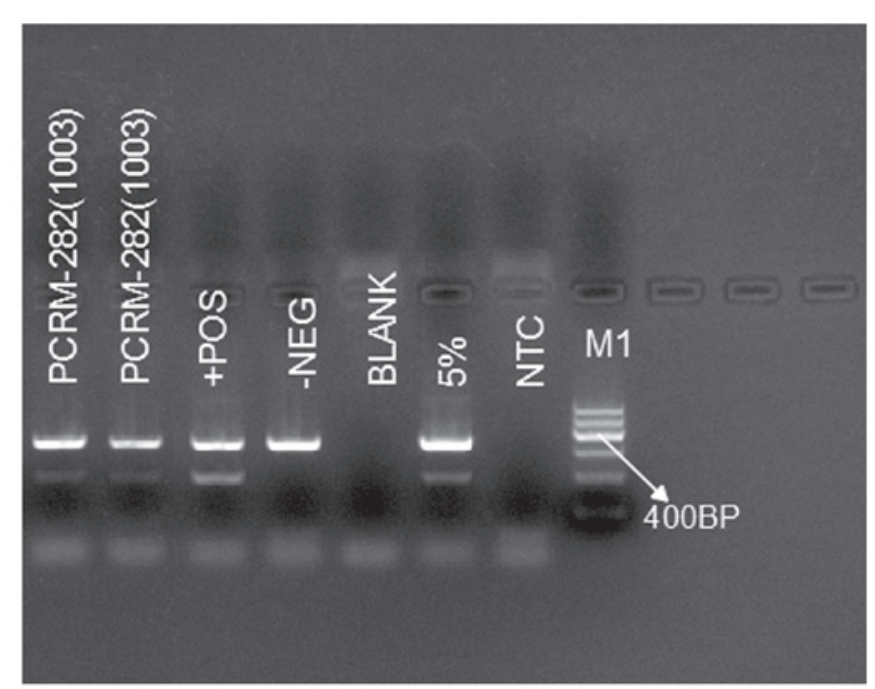

Figure 1. Detection of JAK2-V617F mutation (PCRM-282) + +POS, positive quality control; -NEG, negative quality control; BLANK, blank control; $5 \%$, $5 \%$ detection sensitivity; NTC, reagent control; M1, marker 1.

A

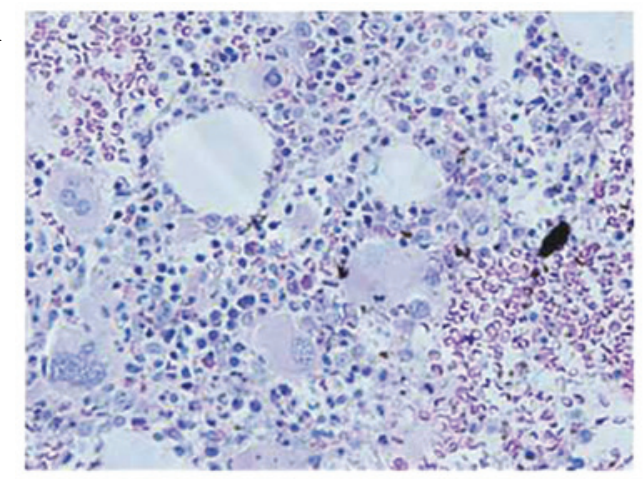

B

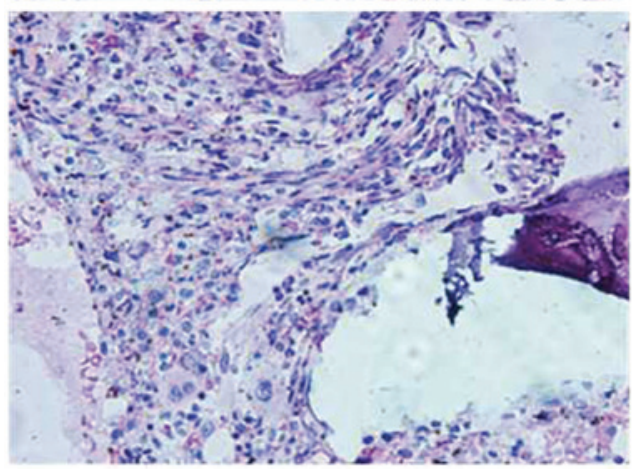

Figure 2. (A) Normal bone marrow biopsy (top). (B) Overactive bone marrow hyperplasia. Focal reticulin fiber hyperplasia, elevated megakaryocyte count, megakaryocytic series dysplasia and pleomorphic changes, and multiple megakaryocyte clusters (hepatocyte growth factor staining; magnification, x400).

hyperplasia, an elevated megakaryocyte count (clusters of megakaryocytes were observed), megakaryocytic series dysplasia and pleomorphic changes, multiple megakaryocyte clusters and focal reticulin fiber hyperplasia (upon plastic embedding and hepatocyte growth factor staining; Fig. 2). This was indicative of a diagnosis of an MPN.

One week later, genetic analysis found the patient to be positive for the JAK2-V617F mutation, and negative for the JAK2 exon 12 and 13 mutations and the BCR-ABL (p210) fusion gene (Fig. 1). T cell subgroup and peripheral blood analyses were normal. The erythrocyte sedimentation rate and autoantibody profile were also normal. Repeat routine blood tests did not show any abnormalities. The final diagnosis was of an unclassifiable MPN. No treatment was administered and the patient was discharged with subsequent monthly follow-up examinations planned. At the time of writing, the patient was in a stable condition.

\section{Discussion}

MPNs, previously categorized under chronic myeloproliferative disorders, are a type of hyperplastic disease of stem cell origin, with heterogenous clinical features and characterized by an increased peripheral blood cell count, overactive bone marrow hyperplasia, and proliferation and differentiation of mature hematopoietic cells (10). MPNs are neoplasms developed from the continuous cloned proliferation of one or multiple series of relatively mature and differentiated bone marrow cells, characterized by increased bone marrow nucleated cells, terminal differentiation and mature proliferative cells, and frequently without dysplasia. MPNs usually have an insidious onset, with one or multiple series of elevated peripheral blood cells, often accompanied by hepatosplenomegaly. Chronic myelogenous MPNs include leukemia, polycythemia vera (PV), essential thrombocythemia (ET), chronic idiopathic myelofibrosis (CMF), chronic neutrophilic leukemia, chronic eosinophilic granulocyte leukemia/hypereosinophilic syndrome, and certain cases of unclassifiable MPN (11-13). $\mathrm{MF}$, bone marrow failure and the development of acute leukemia can be observed in the later stage of MPN. In 2005, multiple studies (11-15) reported a high frequency of point mutations in the JAK2 gene, JAK2-V617F, in cases of MPN and proposed that this mutation may be a unique molecular biomarker of BCR/ABL-negative MPN. This finding has brought about revolutionary progression in the research into the pathogenesis, clinical classification, diagnosis and treatment of MPNs, creating the so-called 'JAK2 era.' It has also been reported that the incidence of the JAK2-V617F mutation in PV, ET and CMF can be as high as 65-97, 23-57 and 35-57\%, respectively $(16,17)$. However, the presence of the JAK2-V617F mutation alone cannot be used to distinguish different categories of MPN, but can be used for an accurate diagnosis when supplemented with bone marrow cytology and bone marrow biopsy histology (18).

Unclassifiable MPN is also termed as undifferentiated MPN, accounting for 10-20\% of MPN cases. Unclassifiable MPN refers to diseases with clinical (hepatosplenomegaly), laboratory (no Ph chromosome and/or BCR-ABL fusion gene) and morphological (leukocytosis, thrombocytosis, with or without anemia, with or without elevated red blood cell count) characteristics of MPN, and does not conform to any diagnostic criteria specific to MPN or include a disease overlap with >2 MPN characteristics. To the best of our knowledge, there have been no previous MPN cases presenting with a normal complete blood cell count. In the present patient, the complete blood cell count was normal, and the patient presented with splenomegaly, tested positive for the JAK2 gene and demonstrated bone marrow pathological characteristics typical of MPN. Therefore, the patient was diagnosed 
with unclassifiable MPN until confirmation could be obtained. However, with no standard guidelines, this neoplasm could not be classified. We propose that the patient's condition may be a precursor of a certain type of MPN, and may later fall into one of the established MPN categories, such as PV, ET or CMF.

The incidence of unclassifiable MPN is low, usually with an occult onset and atypical manifestation. The disease therefore requires special attention in order to avoid misclassification, particularly in patients with normal complete blood cell counts. However, the detection of JAK2 gene mutations may aid the diagnosis, and thus enable the early detection and treatment of unclassifiable MPN cases, and the prevention or reduction in the incidence of thrombosis or hemorrhage. The detection of JAK2 gene mutations is also of great significance in the diagnosis, classification and effective management of MPNs.

In conclusion, MPN with a normal complete blood cell count is rare. IFN- $\alpha$ may aid in the preventention of MPN development; however, more clinical trials are required to confirm this treatment. Recently, due to the identification of the JAK2, MPL and CALR genes diagnosising MPN is easier (19). The case presented in the current study is extremely rare; the clinical manifestation of the present patient is atypical and easy to misdiagnosis. Therefore, unexplained cases of splenomegaly with normal complete blood cell counts should undergo routine bone marrow biopsies and JAK2 gene testing in order to provide the correct diagnosis.

\section{Acknowledgements}

The authors would like to thank Professor Ling Su (Ningxia People's Hospital) for collecting and managing the data for the study.

\section{References}

1. Mahjoub S, Baccouche H, Sahnoun M, Kaabi H, Manai Z, Slama H and Ben Romdhane N: The JAK2 mutation in myeloproliferative neoplasms: A predictive factor of thrombosis. Tunis Med 93: 474-477, 2015.

2. Sørensen AL and Hasselbalch HC: Antecedent cardiovascular disease and autoimmunity in Philadelphia-negative chronicmyeloproliferative neoplasms. Leuk Res pii: S0145-2126, 30555-5, 2015 (Epub ahead of print)

3. Hofmann I: Myeloproliferative neoplasms in children. J Hematop 8: 143-157, 2015.

4. Abelsson J, Andréasson B, Samuelsson J, Hultcrantz M, Ejerblad E, Johansson B, Emanuel R, Mesa R and Johansson P: Patients with polycythemia vera have worst impairment of quality of life among patients with newly diagnosed myeloproliferative neoplasms. Leuk Lymphoma 54: 2226-2230, 2013.
5. Shirane S, Araki M, Morishita S, Edahiro Y, Ohsaka A and Komatsu N: Current problems in the diagnosis of Philadelphia-negative myeloproliferative neoplasms in Japan. Rinsho Ketsueki 56: 877-882, 2015 (In Japanese).

6. Frederiksen H, Farkas DK, Christiansen CF, Larsen TS, Hasselbalch HC, Stentoft J and Sørensen HT: Survival of patients with chronic myeloproliferative neoplasms and new primary cancers: A population-based cohort study. Lancet Haematol 2: e289-296, 2015.

7. Yoshida M, Horiuchi M, Ueda H, Hagihara K, Kanashima H, Nakao $T$, Hirata $C$, Inoue $T$ and Yamane $T$ : Cutaneous extramedullary hematopoiesis associated with myelodysplastic/myeloproliferativeneoplasm, unclassifiable. Rinsho Ketsueki 56: 911-914, 2015 (In Japanese).

8. Kiladjian JJ: Current therapies and their indications for the Philadelphia-negative myeloproliferativeneoplasms. Am Soc Clin Oncol Educ Book 2015: e389-396, 2015.

9. Schmitt-Graeff AH: Chronic myeloid neoplasms. Diagnostic criteria and current therapeutic concepts. Pathologe 31: 29-41, 2010 (In German).

10. Tefferi A and Vardiman JW: Classification and diagnosis of myeloproliferative neoplasms: The 2008 World Health Organization criteria and point-of-care diagnostic algorithms. Leukemia 22: 14-22, 2008.

11. Baxter EJ, Scott LM, Campbell PJ, East C, Fourouclas N, Swanton S, Vassiliou GS, Bench AJ, Boyd EM, Curtin N, et al: Acquired mutation of the tyrosine kinase JAK2 in human myeloproliferative disorders. Lancet 365: 1054-1061, 2005.

12. Jones AV, Kreil S, Zoi K, Waghorn K, Curtis C, Zhang L, Score J, Seear R, Chase AJ, Grand FH, et al: Widespread occurrence of the JAK2 V617F mutation in chronic myeloproliferative disorders. Blood 106: 2162-2168, 2005.

13. Levine RL, Wadleigh M, Cools J, Ebert BL, Wernig G, Huntly BJ, Boggon TJ, Wlodarska I, Clark JJ, Moore S, et al: Activating mutation in the tyrosine kinase JAK 2 in polycythemia vera, essential thrombocythemia and myeloid metaplasia with myelofibrosis. Cancer Cell 7: 387-397, 2005.

14. Kralovics R, Passamonti F, Buser AS, Teo SS, TiedtR, Passweg JR, Tichelli A, Cazzola M and Skoda RC: A gain-of-function mutation of JAK2 in myeloproliferative disorders. N Engl J Med 352: 1779-1790, 2005.

15. James C,Ugo V,Le Couédic JP, Staerk J, Delhommeau F, LacoutC, Garçon L, Raslova H, Berger R, Bennaceur-Griscelli A, et al: A unique clonal JAK2 mutation leading to constitutive signalling causes polycythaemia vera. Nature 434: 1144-1148, 2005.

16. Levine RL and Wernig G: Role of JAK-STAT signaling in the pathogenesis of myeloproliferative disorders. Hematology Am Soc Hematol Educ Program 233-239, 2006.

17. Bumm TG, Elsea C, Corbin AS, Loriaux M, Sherbenou D, Wood L, Deininger J, Silver RT, Druker BJ and Deininger MW: Characterization of murine JAK2V617F-positive myeloproliferative disease. Cancer Res 66: 11156-11165, 2006.

18. Vardiman. J, Brunning RD, Arber DA, et al: Introduction and overview of the classification of the myeloid neoplasms. In: WHO Classification of Tumours of Haematopoietic and Lymphoid Tissues. Swerdlow SH, Campo E, Harris NL, et al (eds). IARC Press, Lyon, France, pp18-30, 2008.

19. Labastida-Mercado N, Galindo-Becerra S, Garcés-Eisele J, Colunga-Pedraza P, Guzman-Olvera V, Reyes-Nuñez V, Ruiz-Delgado GJ and Ruiz-Argüelles GJ: The mutation profile of JAK2, MPL and CALR in Mexican patients with Philadelphia chromosome-negative myeloproliferative neoplasms. Hematol Oncol Stem Cell Ther 8: 16-21, 2015. 